\title{
A method for incorporating organ motion due to breathing into 3D dose calculations in the liver: Sensitivity to variations in motion
}

\author{
Anthony E. Lujan, ${ }^{\text {a) }}$ James M. Balter, and Randall K. Ten Haken \\ Department of Radiation Oncology, University of Michigan, Ann Arbor, Michigan 48109-0010
}

(Received 19 February 2003; revised 30 June 2003; accepted for publication 23 July 2003; published 17 September 2003)

\begin{abstract}
Organ motion has been previously described using a probability distribution function that depends solely upon the amplitude of motion and the degree of asymmetry in the breathing cycle, and that function has been used with patient specific parameters to correct static dose distributions for patient breathing using a dose convolution method. In this study, the consequences of errors in the selection of those two parameters were evaluated. Patients previously treated using a focal liver dose escalation protocol were selected with tumors located in the superior or inferior portion of the liver. For a fixed degree of asymmetry (amplitude), the amplitude (asymmetry) of motion was varied about its nominal value and the consequences of organ motion on the dose distribution and the (potentially new) prescription dose were evaluated. These comparisons show that small $( \pm 3$ $\mathrm{mm}$ ) variations of the amplitude of motion about the nominally measured value may not result in clinically significant changes ( $<$ a single fraction change in the prescription dose), however, larger variations $(>5 \mathrm{~mm})$ can lead to significant changes. Assuming from measurement that the patient breathes asymmetrically (spends more time at expiration), variations in the assumed degree of asymmetry rarely lead to clinically significant changes; the most significant cause for concern being when the patient breathing cycle is maximally different from the treatment planning case (e.g., patient assumed to spend more time at expiration, but later breaths symmetrically). The results point out where quality assurance efforts should be concentrated to help assure the validity of the assumptions used to correct the static dose distributions for patient breathing using the convolution method. (C) 2003 American Association of Physicists in Medicine. [DOI: 10.1118/1.1609057]
\end{abstract}

Key words: organ motion, dose calculations, uncertainty analysis

\section{INTRODUCTION}

A method has previously been presented to incorporate rigidbody organ motion due to breathing into three-dimensional (3D) dose calculations for the treatment of liver disease. ${ }^{1}$ This method is based on the convolution of a static dose distribution with a probability distribution function (PDF) that describes the organ motion due to breathing. Observations of the motion of the diaphragm (and by inference the liver) under fluoroscopy showed that organ motion due to breathing is predominantly in the superior-inferior (SI) direction $^{2-4}$ and is periodic but asymmetric with more time spent near the position at expiration compared to the position at inspiration. In an ultrasound study, Davies et al. ${ }^{3}$ report that the motion of the liver is on average $2 \mathrm{~mm}$ less than the motion of the diaphragm. Korin et al. ${ }^{4}$ found that the predominant motion of the liver was in the SI direction with little expansion or contraction. In the previous study, ${ }^{1}$ the effects of rigid-body organ motion due to breathing on the treatment plan and prescription dose were retrospectively analyzed in the context of a focal liver dose escalation treatment protocol used at the University of Michigan (UM) ${ }^{5-7}$ Patients are CT scanned at the exhale position with asymmetric planning target margins designed for each patient based on the motion observed under fluoroscopy to account for the patient free-breathing during treatment. In that protocol, the prescription (protocol) dose is determined based ${ }^{8}$ on the effective volume ${ }^{9,10}$ of the uninvolved liver. Rigid-body organ motion can be incorporated into the treatment planning dose calculation to mimic potential changes to the dose distribution during treatment (patient breathing freely). The resulting effective volume and hence prescription dose is then determined. Nominal parameters describing the PDF used to model the organ motion (amplitude of motion and degree of asymmetry) are determined from pre-treatment fluoroscopy. The goal of this study is to evaluate the consequence of errors in the selection of those parameters. Potential differences between the nominal parameters and the parameters that may more truly describe the actual motion will be analyzed using the UM protocol for treatment of focal liver tumors.

\section{METHODS AND MATERIALS}

The algorithm for convolving organ motion due to breathing has previously been presented ${ }^{1}$ and is summarized as

$$
\bar{D}(x, y, z)=\int D_{0}\left(x, y, z-z^{\prime}\right) p_{\text {om }}\left(z^{\prime}\right) d z^{\prime},
$$

where $\bar{D}(x, y, z)=$ dose to a point $(x, y, z)$ incorporating the effects of organ motion, $D_{0}\left(x, y, z-z^{\prime}\right)=$ static treatment dose calculation to a point $\left(x, y, z-z^{\prime}\right)$, and $p_{\mathrm{om}}\left(z^{\prime}\right)=\mathrm{PDF}$ that describes organ motion in the SI direction due to breathing.

The PDF is based on a model describing organ motion given by 


$$
z(t)=z_{0}-b \cos ^{2 n}(\pi t / \tau)
$$

where $z(t)=$ position of organ at time $t, z_{0}=$ position at expiration, $b=$ amplitude (extent) of motion, $z_{0}-b=$ position at inspiration, $\tau=$ period of motion, and $n=$ degree of asymmetry (larger $n=$ more time near expiration). The corresponding PDF is given as

$$
\begin{aligned}
p_{\mathrm{om}}(z)= & \left\{n b \pi\left(\frac{z_{0}-z}{b}\right)^{(2 n-1) / 2 n}\right. \\
& \left.\times\left[1-\left(\frac{z_{0}-z}{b}\right)^{1 / n}\right]^{1 / 2}\right\}^{-1} \quad \text { for } z_{0}-b<z<z_{0} .
\end{aligned}
$$

This PDF depends solely on the amplitude of motion and degree of asymmetry. Use of this single PDF in Eq. (1) assumes that patient breathing does not vary over the course of treatment. In reality, the amplitude of patient breathing may vary over time or the patient may occasionally take deep breaths during the course of treatment. Such variations may compromise the selection of a single amplitude for the motion and/or a single degree of asymmetry for the breathing pattern.

As can be seen in Fig. 1, more than one value of $n$ may seem appropriate for a single observation of the breathing pattern ( $n$ is restricted to integer values). Alternatively, a patient may breathe in a quite different manner during treatment compared to the manner observed under treatment planning conditions. However in practice, a nominal parameter set (amplitude of motion and degree of asymmetry) is usually selected for a patient simulation to predict the effects of the organ motion due to breathing over the projected course of treatment.

Here, the computationally fast convolution method (using the initial-nominal breathing parameters) is compared to direct simulations ${ }^{1,11,12}$ of expected patient outcomes (allowing for deviations and/or variations from the initial parameter set) to identify the sensitivity of the single observation convolution method to changes in the breathing parameters. This direct simulation method is summarized in the following.

(1) The organ displacement (away from the nominal expiration position) due to breathing as a function of time was separated into 10 discrete evenly spaced (in distance) bins. The time-weighted average organ position in each bin was computed using the amplitude $b$, and degree of asymmetry $n$ specified for any particular simulation.

(2) The change in organ position was simulated in the treatment planning system by moving the beams and isocenter in a direction opposite to the motion.

(3) Monitor units were held constant to compute dose at other positions in the respiratory cycle.

(4) The dose distribution was recalculated for each position simulated.

(5) The probability $p_{b i}$ of finding the organ in the $i$ th bin based on the motion described with Eq. (2) was computed.

(6) Each calculation was weighted by $p_{b i}$. a) $n=3$

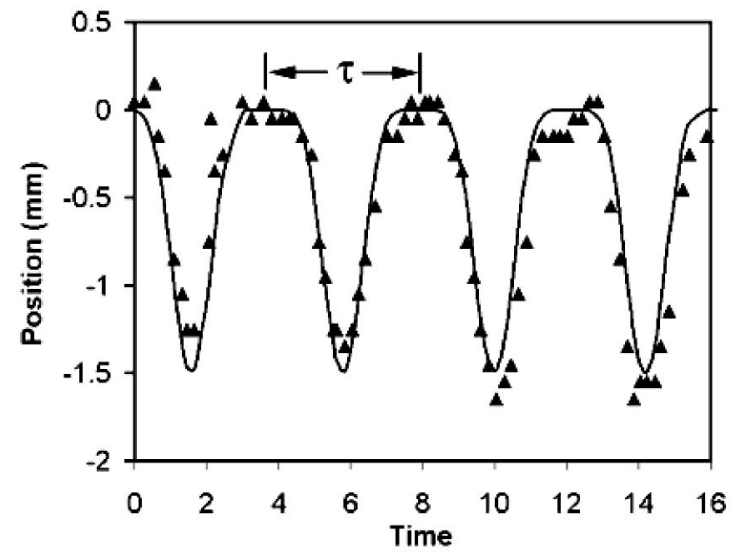

b) $n=2$

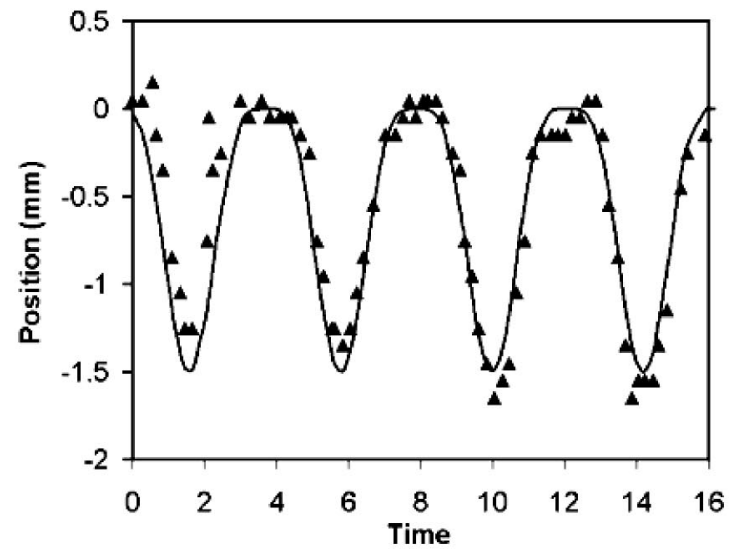

c) $n=6$

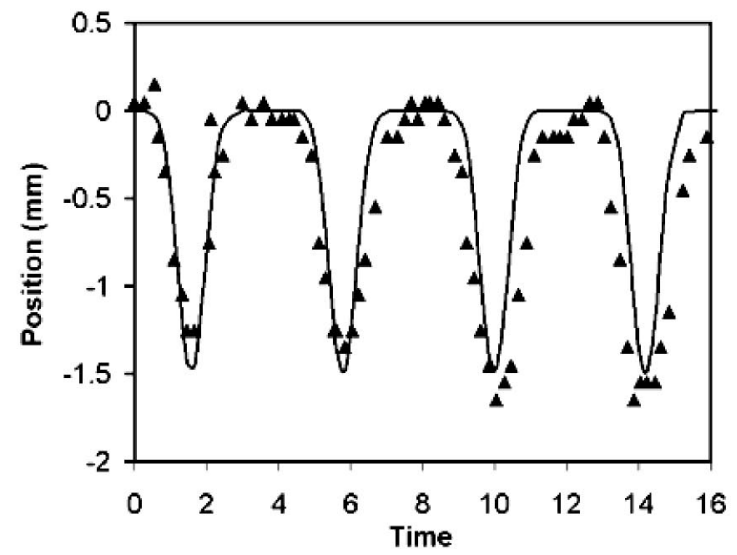

FIG. 1. Measured time course of breathing for a sample patient based on diaphragm motion (away from the expiration position) as observed under fluoroscopy compared to model given by Eq. (2) using (a) $n=3$, (b) $n=2$, and (c) $n=6$.

(7) Individual simulations were summed to form a composite dose distribution using the original treatment planning geometry as a backdrop.

In this study, nominal values of $b=1.5 \mathrm{~cm}$ (amplitude of 
motion) and $n=3$ (degree of asymmetry) were selected based on general observations of diaphragmatic movement under fluoroscopy for many patients.

\section{A. Variations in the amplitude of motion}

For a fixed degree of asymmetry $(n=3)$, the amplitude of motion was varied from $b=1.2 \mathrm{~cm}$ to $b=1.8 \mathrm{~cm}$ (in $0.1 \mathrm{~cm}$ increments) and the probability per bin, $p_{b i}(b, n=3)$ was calculated. Direct simulations of the organ motion for each $b$ (assumed to remain constant over the course of treatment) were performed as described earlier. Deep-breath conditions were also simulated for $b=2.0,2.5$, and $3.0 \mathrm{~cm}$. Two occasional deep-breath scenarios were calculated to simulate the effect of a patient breathing normally $(b=1.5 \mathrm{~cm})$ for $75 \%$ of the treatment while taking a deep breath $(b=3.0 \mathrm{~cm}) 25 \%$ of the time (henceforth referred to as db1), and a simulation was performed to show the effects of a patient taking a deep breath $(b=3.0 \mathrm{~cm})$ only $10 \%$ of the treatment time and breathing normally for the remainder of the treatment (referred to as $\mathrm{db} 2$ ).

Next, a treatment was simulated for $b=1.2 \mathrm{~cm} 50 \%$ of the time, and $b=1.8 \mathrm{~cm} 50 \%$ of the time (referred to as avg 1). For each of the above-mentioned simulations, values for $p_{b i}(b, n=3)$ were calculated and the dose distributions were re-calculated using our direct simulation.

\section{B. Variations in the asymmetry of the breathing cycle}

For a fixed value of $b=1.5 \mathrm{~cm}$, the degree of asymmetry was varied from $n=1$ (symmetric periodic function) to $n$ $=10$ (highly asymmetric with approximately $70 \%$ of the time spent near the expiration position) $(n=3$ is the nominal value). We also performed simulations for $n=0$ (patient near the inspiration position for the entire treatment), $n=50$ (approximately $86 \%$ of the time spent near the expiration position), and $n=100$ (approximately $90 \%$ of the time spent near the expiration position). For each $n, p_{b i}(n, b=1.5 \mathrm{~cm})$ was calculated and the corresponding dose distribution computed using our direct simulation procedure.

\section{Clinical assessment of dose distribution simulations}

For each dose distribution calculated, the effective volume of the uninvolved liver (liver minus gross tumor volume) was computed and the corresponding prescription dose (assigned by the physician based on the protocol) was determined based upon iso-complication probability curve $(10 \%$ risk of complication) for the liver as shown in Fig. $2 .{ }^{6}$ The prescription dose is incremented in 1.5 Gy steps and rounded to the lower 1.5 gray fraction with a maximum prescription dose of $90 \mathrm{~Gy}$, and a minimum dose of $36 \mathrm{~Gy}$ (corresponding to a $V_{\text {eff }}$ of $0.90 \%$ ) depending on the risk level.

\section{Patient analysis}

Ten patient geometries were simulated as described earlier for this study. All patients were treated on clinical trials ${ }^{6,7}$ after informed consent was obtained in accordance with the procedures of the Institutional Review Board (IRB) of the

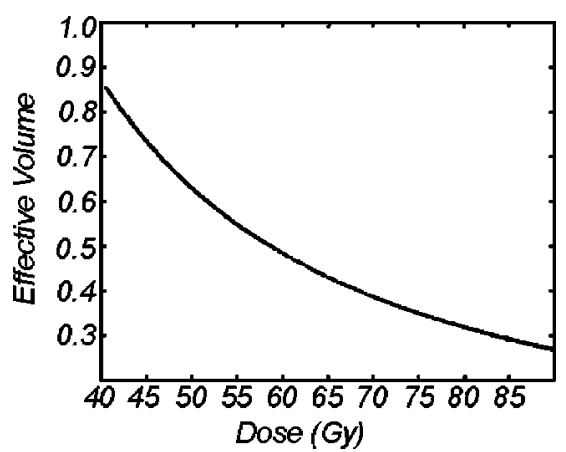

FIG. 2. Iso-complication probability curve for the liver for $10 \%$ risk level as a function of prescription dose (Ref. 8).

University of Michigan, and CT-scanned for treatment planning with voluntary breath-hold at normal expiration. ${ }^{2} \mathrm{Tu}-$ mor volumes were located in the upper (superior) portion of the liver (5 patients) or in the inferior portion of the liver (5 patients). Planning target volume (PTV) margins were originally designed individually for each patient using their measured breathing excursion inferiorily. ${ }^{2}$ However, to normalize the results of the simulations here, we assumed each patient's nominal treatment plan included a standard $15 \mathrm{~mm}$ of excursion for breathing $(b=1.5 \mathrm{~cm})$. The nominal prescription dose (again, as assigned by the physician based on the protocol) was determined for each patient based on this nominal $15 \mathrm{~mm}$ excursion for breathing. All patients were planned using our in-house treatment planning software (UMPLANUniversity of Michigan, Ann Arbor).

\section{RESULTS}

Table I summarizes the changes in effective volume due to variations in the amplitude of motion $(b)$, deep breath scenarios (db 1 and db 2), the average simulation (avg 1) as well as changes in asymmetry $(n)$ described earlier for representative patients with tumors located in the superior ( $\mathrm{I} a$ ) and inferior $(\mathrm{I} b)$ portions of the liver, respectively. The nominal simulation values $(n=3, b=1.5 \mathrm{~cm})$ are indicated as are the values for the static $(b=0)$ treatment plan (no accounting for dose changes due to breathing).

For each patient studied, there is a change in the effective volume of the uninvolved liver when the effects of organ motion due to breathing are included in the dose calculation. In all cases (CT scan performed at expiration) regions superior to the tumor volume move into the beam and regions inferior to the tumor move out of the beam as the patient breathes. For patients 1-5 with tumors located in the superior portion of the liver, organ motion due to breathing results in a decrease in the effective volume compared to the static $(b=0)$ situation. For patients with tumors in the inferior portion of the liver, the effective volume will increase as the patient breathes. These changes in $V_{\text {eff }}$ determined prior to treatment may have resulted in the physician making changes to the prescription dose if they had known this prior to treatment. ${ }^{13}$ For treatment geometries in which the tumor is located in the middle portion of the liver (simulations not included here), the overall volume of the liver that is exposed 
TABLE I. Effective volume of the uninvolved liver for two representative patients; one with a tumor located in the superior $(\mathrm{I} a)$ portion of the liver and one with a tumor located in the inferior $(\mathrm{I} b)$ portions of the liver. $V_{\mathrm{eff}}$ as a function of $b$ for a fixed degree of asymmetry $(n=3)$ and $V_{\text {eff }}$ as a function of $n$ for a fixed amplitude of motion $(b=1.5 \mathrm{~cm})$ are shown. Nominal values $(b=1.5 \mathrm{~cm}, n=3)$ are indicated. Key: db1 = simulation of patient breathing with nominal amplitude $75 \%$ of time, and deep breath $(b$ $=3.0 \mathrm{~cm}) 25 \%$ of the time, $\mathrm{db} 2=$ simulation of patient breathing with nominal amplitude $90 \%$ of the time and deep breath $10 \%$ of the time, avg1 $=$ simulation of patient breathing with $b=1.2 \mathrm{~cm} 50 \%$ of the time, and $b$ $=1.8 \mathrm{~cm} 50 \%$ of the time.

\begin{tabular}{|c|c|c|c|c|}
\hline & $b(\mathrm{~cm})$ & $V_{\text {eff }}(\%)$ & $n$ & $V_{\text {eff }}(\%)$ \\
\hline \multirow[t]{14}{*}{ (a) } & 0.0 & 37.92 & 0 & 26.77 \\
\hline & 1.2 & 34.91 & 1 & 31.90 \\
\hline & 1.3 & 34.69 & 2 & 33.27 \\
\hline & 1.4 & 34.36 & 3 & 34.07 \\
\hline & 1.5 & 34.07 & 4 & 34.53 \\
\hline & 1.6 & 33.80 & 5 & 34.80 \\
\hline & 1.7 & 33.49 & 6 & 35.00 \\
\hline & 1.8 & 33.20 & 7 & 35.23 \\
\hline & 2.0 & 32.63 & 8 & 35.39 \\
\hline & 2.5 & 31.17 & 9 & 35.57 \\
\hline & 3.0 & 29.69 & 10 & 35.58 \\
\hline & $\mathrm{db} 1$ & 32.95 & $\infty$ & 37.92 \\
\hline & $\mathrm{db} 2$ & 33.62 & & \\
\hline & avg 1 & 34.05 & & \\
\hline \multirow[t]{14}{*}{ (b) } & 0.0 & 38.26 & 0 & 49.09 \\
\hline & 1.2 & 40.99 & 1 & 43.43 \\
\hline & 1.3 & 41.09 & 2 & 41.88 \\
\hline & 1.4 & 41.22 & 3 & 41.32 \\
\hline & 1.5 & 41.32 & 4 & 40.90 \\
\hline & 1.6 & 41.44 & 5 & 40.57 \\
\hline & 1.7 & 41.53 & 6 & 40.30 \\
\hline & 1.8 & 41.60 & 7 & 40.21 \\
\hline & 2.0 & 41.70 & 8 & 40.08 \\
\hline & 2.5 & 41.81 & 9 & 40.04 \\
\hline & 3.0 & 41.63 & 10 & 39.84 \\
\hline & $\mathrm{db} 1$ & 41.30 & $\infty$ & 38.26 \\
\hline & $\mathrm{db} 2$ & 41.30 & & \\
\hline & avg 1 & 41.26 & & \\
\hline
\end{tabular}

to the high dose region of the beam will change relatively little, although some change is expected due to the asymmetric shape of the liver itself.

\section{A. Variations in the amplitude of motion}

As the amplitude (extent) of motion varies in a narrow range $(b=1.2$ to $b=1.8 \mathrm{~cm})$ about the nominal $(b=1.5)$ value, the effective volume of the normal liver only changes slightly (middle portion of Fig. 3). However, in all cases the magnitude of the variation is less than the original change from the static $(b=0)$ case obtained by accounting for the average motion in the first place.

For all the patients severe over-estimation of the amplitude at planning ( $b \rightarrow 0$ relative to the assumed $b=1.5 \mathrm{~cm}$ ) leads to large changes in $V_{\text {eff }}$ (far left portion of Fig. 3). For larger under-estimations of the amplitude at planning $(b$ $=2.0$ to $b=3.0 \mathrm{~cm}$ relative to the assumed $b=1.5 \mathrm{~cm})$, the change in $V_{\text {eff }}$ relative to the nominal value will increase for tumors located in the superior portion of the liver (far right portion of Fig. 3). These variations in $V_{\text {eff }}$ about the nominal

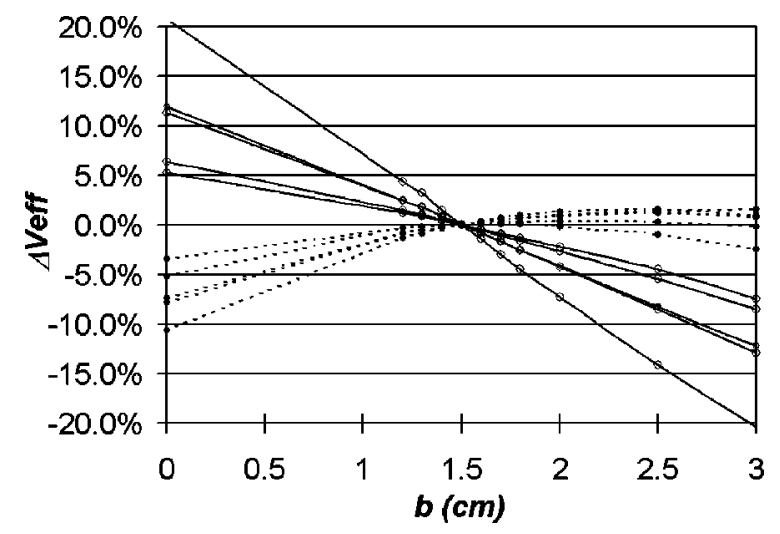

FIG. 3. Change in $V_{\text {eff }}$ from the nominal value computed for $b=1.5 \mathrm{~cm}$ and $n=3$ as a function of $b$. Solid line and open circles represent patients $1-5$ (tumors in the superior portion of the liver), dashed line and closed circles represent data for patients 6-10 (tumors in the inferior portion of the liver).

value can also lead to changes in the prescription dose in our protocol setting.

The above-mentioned results assume that the patient breathes with the same (but different from that originally planned) magnitude throughout the entire course of treatment, and represent potential worst-case scenarios. If the amplitude of breathing varies over the course of treatment, a situation such as that presented in avg 1 may result $(50 \%$ of the treatment spent at extremes about the nominal position, $b=1.2$ and $b=1.8 \mathrm{~cm})$. The value of $V_{\text {eff }}$ will change from the nominal value (Table I), but the change may not be sufficient to warrant a change from the nominal prescription dose.

In situations where the patient occasionally takes deep breaths over the course of treatment ( $\mathrm{db} 1, \mathrm{db} 2)$, the effective volume also changes from the nominal value (Table I). However, if a patient takes a deep breath even $25 \%$ of the time during the course of treatment, there will likely only be a single fraction change in the protocol dose ( $\mathrm{db} 1)$ and for a $10 \%$ deep breath simulation ( $\mathrm{db} 2$ ), potentially no change in the prescription dose.

\section{B. Variations in the asymmetry of the breathing cycle}

The results of the studies of variations in the asymmetry $n$ are also summarized in Table I and Fig. 4, where $n=\infty$ represents the static treatment plan (no motion). As the degree of asymmetry increases ( $n$ increases), the amount of time a patient spends near the expiration position will increase, and as would be expected, the effective volume of the uninvolved liver approaches the value of $V_{\text {eff }}$ for the static $(b=0)$ treatment plan that did not account for motion (again, patients were scanned near the expiration position). This asymptotic trend is observed by $n=10$ as seen in Fig. 4 hence, the data for $n=50, n=100$ are not shown. For $n=0$ (patient treated near the inspiration position), there is a large difference between the effective volume predicted using our nominal value $n=3$ and the effective volume resulting from $n=0$ (far left portion of Fig. 4). This is consistent with the patient treatment position being maximally different from both the 


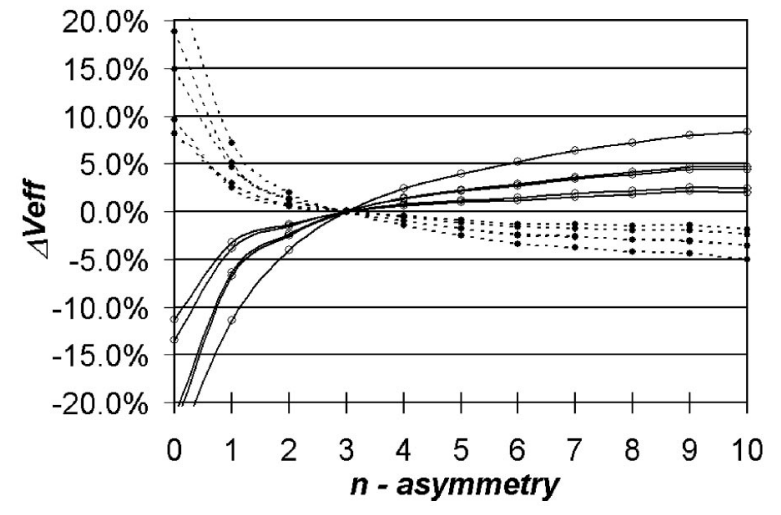

FIG. 4. Change in $V_{\text {eff }}$ from the nominal value computed for $b=1.5 \mathrm{~cm}$ and $n=3$ as a function of $n$. Solid line and open circles represent patients 1-5 (tumors in the superior portion of the liver), dashed line and closed circles represent data for patients 6-10 (tumors in the inferior portion of the liver). Data at $n=10$ begin to demonstrate asymptotic approach to $n=\infty$ value.

static (expiration) treatment planning position and from any situation in which the patient breathes freely. In all other cases, the largest remaining difference in $V_{\text {eff }}$ (relative to the nominal case) is for the patient breathing in a completely symmetric pattern $(n=1)$ when an asymmetric breathing pattern $(n=3)$ had been assumed. The resultant changes in the effective volume are, in many cases, sufficient to justify a change in the protocol dose to maintain a fixed risk of complication. The effective volumes change in manners consistent with the above-noted observations for the superior and inferior tumor geometries.

\section{DISCUSSION}

Intra-treatment organ motion due to breathing can lead to erroneous prediction of the dose delivered to a patient when a computed tomography (CT) scan of a patient is used for treatment planning dose calculations (even in cases where the CT is obtained with a patient breath-hold at inspiration or expiration) if treatment is not also delivered accordingly. Previously, we proposed a simple model to describe the organ motion due to breathing that depends upon specification of the extent of motion $b$, and the asymmetry of the breathing cycle $n$ (parameters are selected from pre-treatment fluoroscopy). Here, we have investigated the effects that potential errors in the selection of these parameters may have on the resultant dose distribution by examining variations in the parameters about nominal values.

As the amplitude (extent) of motion varies about the nominally observed value, $V_{\text {eff }}$ will also vary (which may, in turn, effect the selection of the prescription dose in our protocol). The magnitude of the change in $V_{\text {eff }}$ required to affect a change in the protocol dose (relative to the prescription dose in the nominal setting) is a function of the nominal value of $V_{\text {eff }}$.

It is possible to estimate the magnitude of change in $V_{\text {eff }}$ that would be sufficient to result in a change in the prescription dose. The scale of the prescription dose is divided into evenly spaced dose bins of $1.5 \mathrm{~cm}$. If we examine the iso-
NTCP curve (Fig. 2) used to determine the prescription dose (based on $V_{\text {eff }}$ ), we see that for small values of $V_{\text {eff }}(<0.40)$, approximately a $2.5 \%$ change in the effective volume will result in a single fraction $(1.5 \mathrm{~Gy})$ change in the prescription dose. For medium and high $V_{\text {eff }}$ values $(>0.40)$, changes in the prescription dose (in 1.5 Gy steps) are associated with $3 \%-6 \%$ changes in $V_{\text {eff }}$. The percent change in $V_{\text {eff }}$ (relative to the nominal value) is shown in Fig. 3 for changes in the extent of motion from $b=0 \mathrm{~cm}$ to $b=3.0 \mathrm{~cm}$ relative to the nominal $b=1.5 \mathrm{~cm}$ case.

If we examine small $(b=1.2 \mathrm{~cm}$ to $b=1.8 \mathrm{~cm})$ variations about the nominal value (changes that might be associated with systematic error in the original measurement of amplitude), we see that the percent change in $V_{\text {eff }}$ is small (less than 3\%) compared to the original change in $V_{\text {eff }}$ from the static treatment case $(b=0 \mathrm{~cm})$. These variations are generally larger for tumors located in the superior portion of the liver. However, the magnitude of these changes is, in most cases, only sufficient to warrant at most a single fraction (1.5 Gy) change in the prescription dose from the nominal value. Similar changes in $V_{\text {eff }}$ are seen for our average simulation avg 1 in Table I.

Again, specific results will depend upon the exact treatment geometry as well as the PTV margins selected for the treatment. While the relative change in $V_{\text {eff }}$ is small for small variations in the amplitude of motion about our nominal value, the change in $V_{\text {eff }}$ is potentially large (up to $15 \%$ ) for large changes $(b=0$, or $b=2.0$ to $b=3.0 \mathrm{~cm})$. These larger changes may result in more than a single fraction change in the prescription dose. These deep-breath $(b=2.0$ to $b$ $=3.0 \mathrm{~cm})$ or no-breath $(b=0)$ simulations assume that the patient would breathe with fixed, but incorrect (relative to the nominal parameters selected during treatment planning), amplitude over the entire course of treatment. In an actual treatment, it is more realistic to assume that the average amplitude of the patient's breathing is relatively constant (e.g., $b=1.5 \mathrm{~cm}$ ) but the patient may take occasional deep breaths during the course of treatment. Our simulations (Table I) of these more realistic situations ( $\mathrm{db} 1$ and $\mathrm{db} 2$ ) show that if a patient takes a deep breath for even up to $25 \%$ of the treatment, the change in $V_{\text {eff }}$ (relative to the nominal value) is only sufficient to warrant at most a single fraction change in the prescription dose. If the patient should breathe deeply for only $10 \%$ of the treatment, the change in $V_{\text {eff }}$ may be insufficient to warrant any additional change in the prescription dose (from the nominal value).

The asymmetry of a patient's breathing cycle may also vary over the course of treatment, and the magnitude of this variation is worth considering in the context of our model. The motion of a patient's diaphragm (as observed under fluoroscopy) is shown in Fig. 1 along with our mathematic model. Figure 1(a) shows the same measured data along with our model using $n=3$, while Fig. 1(b) shows the measured data with our model using $n=2$. If we compare this to Fig. $1(\mathrm{c})$, which shows the measured patient data along with our model using $n=6$, we see that it is reasonable to assume that any of these values of $n$ would be sufficient to adequately describe the time course of breathing (recall, our choice of $n$ 
is limited to integer values). These observations highlight the importance of observing a patient breathe over many cycles to provide enough data to simplify our choice of both $n$ and $b$.

The percent change in $V_{\text {eff }}$ as a function of $n$ (relative to the nominal value of $n=3$ ) is shown in Fig. 4. From the data, it is clear that the largest change in $V_{\text {eff }}$ occurs for $n$ $=0$. This is entirely consistent, as for the $n=0$ scenario the treatment takes place with the patient near the inspiration position in the breathing cycle $100 \%$ of the time, a situation completely counter to the treatment planning (scan at expiration) situation. As the degree of asymmetry increases from the assumed value, the value of $V_{\text {eff }}$ begins to slowly approach the static (expiration) simulation geometry $(n=\infty)$.

Assuming that the patient breathes freely over the course of treatment, the largest remaining differences are for simulations where the patient breathes symmetrically $n=1$ when an asymmetric breathing pattern $(n=3)$ had been assumed. These changes in $V_{\text {eff }}$ could be large enough to cause a one or more fraction change in the prescription dose. However, for an asymmetric breathing cycle, our results indicate that our model is relatively insensitive to small changes in the degree of asymmetry. Further, these small changes in the asymmetry would be generally insufficient to result in variations in $V_{\text {eff }}$ large enough to change the prescription dose.

If we are interested in predicting the dose delivered during the course of treatment, careful observations of the nature of organ motion will be required. For example, our results indicate that our prediction of $V_{\text {eff }}$ is sensitive to the amplitude of motion $b$ that we select to model the patient's breathing cycle. Therefore, for an accurate prediction of the dose distribution, it is important to observe and sample the amplitude of the breathing cycle for each patient to determine a proper magnitude for the parameter $b$. Further, if in our observations, we find that the extent of motion for a patient varies greatly, additional efforts may be required to control or monitor the patient's breathing cycle. ${ }^{14-18}$ However, if the amplitude of the breathing motion varies about some nominal value, it is likely that the prescription dose will not change from that selected using that nominal value of $b$ (note: in Fig. 3 the value of $V_{\text {eff }}$ changes sign about the nominal value for positive or negative changes; thus variations that might be associated with random changes in the breathing amplitude would by and large cancel out).

Similarly, we must also be aware of the degree of asymmetry in a patient's breathing cycle for an accurate prediction of the dose distribution. Though our predictions of $V_{\text {eff }}$ are less sensitive to minor changes in the degree of asymmetry for $n>2$, there is a larger change between a purely symmetric breathing cycle $(n=1)$ and a breathing cycle that includes any degree of asymmetry. Hence, if we begin to use methods that cause a patient to breathe in a symmetric pattern, our values selected for $n$ must reflect this change as well. As expected, the worst possible scenario is one in which patients do not breathe freely during the treatment but instead hold their breath near positions far away from where they were treatment planned (e.g., scanned at expiration, but treated at inspiration). In those cases, the effective volume calculated for the static geometry or for free breathing simulations will greatly differ from the actual dose delivered (up to $25 \%$ differences in $V_{\text {eff }}$ values between $n=0$ and $n=3$ ). Again, in such cases, special techniques may be required.

Finally, as the nature of the organ motion is better understood and parametrized, confirmation of convolution-based procedures to simplify the analysis of organ motion due to breathing will require direct simulations that include the effects of changes in the patient external contour, any organ motion that may be present, organ deformation, ${ }^{19}$ and any inhomogeneity corrections that may be required. ${ }^{19,20}$ In addition, a more complex PDF may be required to model the more complex motion. We have presented here a basic framework to verify convolution-based studies.

\section{ACKNOWLEDGMENT}

Work supported by NIH Grant No. P01-CA59827.

${ }^{a}$ Present address: Department of Radiation and Cellular Oncology, University of Chicago, Chicago, Illinois 60637; electronic mail: alujan@uchicago.edu

${ }^{1}$ A. E. Lujan, E. W. Larsen, J. M. Balter, and R. K. Ten Haken, “A method for incorporating organ motion due to breathing into 3D dose calculations," Med. Phys. 26, 715-720 (1999).

${ }^{2}$ J. M. Balter, K. L. Lam, C. J. McGinn, T. S. Lawrence, and R. K. Ten Haken, "Improvement of CT-based treatment-planning models of abdominal targets using static exhale imaging," Int. J. Radiat. Oncol., Biol., Phys. 41, 939-943 (1998).

${ }^{3}$ S. C. Davies, A. L. Hill, R. B. Holmes, M. Halliwell, and P. C. Jackson, "Ultrasound quantitation of respiratory organ motion in the upper abdomen,” Br. J. Radiol. 67, 1096-1102 (1994).

${ }^{4}$ H. W. Korin, R. L. Ehman, S. J. Riederer, J. P. Felmlee, and R. C. Grimm, "Respiratory kinematics of the upper abdominal organs: A quantitative study," Magn. Reson. Med. 23, 172-178 (1992).

${ }^{5}$ T. S. Lawrence, R. K. Ten Haken, M. L. Kessler, J. M. Robertson, J. T. Lyman, M. L. Lavigne, M. B. Brown, D. J. DuRoss, J. C. Andrews, W. D. Ensminger, and A. S. Lichter, "The use of 3D dose volume analysis to predict radiation hepatitis," Int. J. Radiat. Oncol., Biol., Phys. 23, 781788 (1992).

${ }^{6}$ C. J. McGinn, R. K. Ten Haken, W. D. Ensminger, S. Walker, S. Wang, and T. S. Lawrence, "The treatment of intraheptatic cancers with radiation doses based on a normal tissue complication probability model," J. Clin. Oncol. 16, 2246-2252 (1998).

${ }^{7}$ L. A. Dawson, C. McGinn, D. Normolle, R. K. Ten Haken, W. Ensminger, and T. S. Lawrence, "Escalated focal liver radiation and hepatic artery fluorodeoxyuridine for unresectable liver malignancies," J. Clin. Oncol. 18, 2210-2218 (2000).

${ }^{8}$ R. K. Ten Haken, M. K. Martel, M. L. Kessler, M. B. Hazuka, T. S. Lawrence, J. M. Robertson, A. T. Turrisi, and A. S. Lichter, "Use of $V_{\text {eff }}$ and iso-NTCP in the implementation of dose escalation protocols," Int. J. Radiat. Oncol., Biol., Phys. 27, 689-695 (1993).

${ }^{9}$ G. J. Kutcher and C. Burman, "Calculation of complication probability factors for non-uniform normal tissue irradiation: The effective volume method,” Int. J. Radiat. Oncol., Biol., Phys. 16, 1623-1630 (1989).

${ }^{10}$ G. J. Kutcher, C. Burman, L. Brewster, M. Goitein, and R. Mohan, "Histogram reduction method for calculating complication probabilities for three-dimensional treatment planning evaluations," Int. J. Radiat. Oncol., Biol., Phys. 21, 137-146 (1991).

${ }^{11}$ J. H. Killoran, H. M. Kooy, D. J. Gladstone, F. J. Welte, and C. J. Beard, "A numerical simulation of organ motion and daily setup uncertainties: Implication for radiation therapy," Int. J. Radiat. Oncol., Biol., Phys. 37, 212-221 (1997).

${ }^{12}$ J. Leong, "Implementation of random positioning errors in computerized radiation treatment planning systems as a result of fractionation," Phys. Med. Biol. 32, 327-334 (1987).

${ }^{13}$ M. Rosu, J. Balter, D. McShan, L. Dawson, and R. K. Ten Haken, "Alterations in normal liver doses due to patient motion," Med. Phys. 29, 1338 (2002). 
${ }^{14}$ J. W. Wong, M. B. Sharpe, D. A. Jaffray, V. R. Kini, J. M. Robertson, J. S. Stromberg, and A. A. Martinez, "The use of active breathing control (ABC) to reduce margin for breathing motion," Int. J. Radiat. Oncol., Biol., Phys. 44, 911-919 (1999).

${ }^{15}$ H. D. Kubo, P. M. Len, S. Minohara, and H. Mostafavi, "Breathingsynchronized radiotherapy program at the University of California Davis Cancer Center," Med. Phys. 27, 346-353 (2000).

${ }^{16}$ S. Shimizu, H. Shirato, S. Ogura, H. Akita-Dosaka, K. Kitamura, T. Hishioka, K. Kagei, M. Nishimura, and K. Miyasaka, "Detection of lung tumor movement in real-time tumor-tracking radiotherapy," Int. J. Radiat. Oncol., Biol., Phys. 51, 304-310 (2001).

${ }^{17}$ L. A. Dawson, K. K. Brock, S. Kazanjian, D. Fitch, C. J. McGinn, T. S. Lawrence, R. K. Ten Haken, and J. M. Balter, "The reproducibility of organ position using active breathing control $(\mathrm{ABC})$ during liver radiotherapy,” Int. J. Radiat. Oncol., Biol., Phys. 51, 1410-1421 (2001).

${ }^{18}$ J. M. Balter, K. K. Brock, D. W. Litzenberg, D. L. McShan, T. S. Lawrence, R. K. Ten Haken, C. J. McGinn, K. L. Lam, and L. A. Dawson, "Daily targeting of intrahepatic tumors for radiotherapy," Int. J. Radiat. Oncol., Biol., Phys. 52, 266-271 (2002).

${ }^{19}$ K. K. Brock, D. L. McShan, R. K. Ten Haken, S. J. Hollister, L. A Dawson, and J. M. Balter, "Inclusion of organ deformation in dose calculations," Med. Phys. 30, 290-295 (2003).

${ }^{20}$ I. J. Chetty, M. Rosu, N. Tyagi, L. H. Marsh, D. L. McShan, B. A. Fraass, and R. K. Ten Haken, "A fluence convolution method to account for respiratory motion in $3 \mathrm{~d}$ dose calculations of the liver," Med. Phys. (to be published). 